\title{
Hierarchies of adaptable learning during product development
}

\author{
Anna P. Chatzimichali \\ Department of Educational Research and Development \\ School of Business and Economics \\ Maastricht University \\ Maastricht, The Netherlands \\ a.chatzimichali@maastrichtuniversity.nl
}

\author{
Vassilios D. Tourassis \\ Production Engineering and Management Department \\ Faculty of Engineering \\ Democritus University of Thrace \\ Xanthi, Greece \\ vtourasi@pme.duth.gr
}

\begin{abstract}
Shorter product lifecycles, shrinking time-to-market and increasing global competition, drive companies to premature transitions from the development laboratory to full-scale commercial production. This ramp-up period is usually considered as a transient phenomenon and often ignored by a large body of literature. Hence, the current push for accelerated development and quality manufacturing of new products, has increased the need to model and measure production performance during ramp-up. Despite this need for a concrete framework of these early stages of the product life cycle, a useful model of rampup, formalizing this tradeoff between product design and process modeling during the execution phase, is missing. In this context the present work deals with this issue throught a structured methodology that highlights the system sensitivities by decoupling process and product design, proposing an algorithm that uses empirical evaluation measures of manufacturing complexity.

Index Terms-Ramp-up, New Product Development, Learning curve.
\end{abstract}

\section{INTRODUCTION}

In the primary phases of product development, when the production process is not established, many people are involved with different attitudes, competencies and agendas working in different contexts. During this stage, the plant relies on product designers to plan production or organize process equipment. However, considering how inherently dissimilar are the activities of product design and process execution, there is usually a gap causing dysfunctions. Consequently, in a typical product development setting, there are few information pathways between product design and process execution and inadequate tools to surpass arising problems. It is therefore not surprising that to reserve a set of options for future changes, many flexible decisions are made due to narrow product development deadlines. In this sense, flexibility is inherently built into a process developed to handle product variety with less cost and more speed. Leaving many options open, however, has consequences in the production performance, since emerging problems are solved with quick patches and late fixes at the execution phase. Additionally, during ramp-up experienced engineers often employ intuitive methods to solve pre-production problems and act according to their instinct to make cost-effective changes, to improve the manufacturability and overall quality.
In this framework, and while recent efforts have been focused on predicting component quality levels through variability and process capability measures at the design stage, the variability associated with assembly operations is rarely considered in the context of quality [1]. Despite well-documented articles on the functional performance of components (effects of material on process capability, geometric constraints, process precision etc.) [2] there is no work on the relationship between the rate of production ramp-up and product design decision making [3].

To fill this gap, in this paper proposes a dynamic approach with information pathways that take under consideration not only the design and process modifications that occur during ramp-up, but also the learning effects that appear in the early production stages. This approach builds upon two significant observations. First that "while a considerable body of research has focused on the time-to-market and process improvement/development problems in isolation, consideration of both design and production decisions to support bringing new products to market has remained relatively unexplored" [4] and second, that "various techniques have been used to balance product and process design and other manufacturing activities but typically in an unscientific manner" [5, p. 426].

The present work deals with this issue throught a structured methodology that highlights the system sensitivities by decoupling process and product design, and uses proven evaluation measures of manufacturing complexity. Additionally, despite the fact that learning precedes understanding, there are no other known studies combining learning curves of manufacturing systems with complexity.

\section{BACKGROUND THEORY}

\section{A. Investment to learning}

Most models that utilize the learning curve theory usually treat learning as a by-product of cumulated production volume. However, learning should be treated more as a "scarce firmspecific resources that a firm allocates towards the improvement of the cost, quality or timeliness of its existing products and processes" [6]. Relevant studies that support this point of view are summarized in this subsection. 
Research by [7] revealed that investment in induced learning is regarded as a capital investment that yields benefits over multiple periods. Findings suggest that this investment in the removal of defects whenever they occur have a higher impact until a certain quality level is reached. After that, the benefit of improving the quality is not large enough to justify the cost. In the same line of research, an exploratory study in two ship building companies was presented by [8]. The study investigates the investments patterns of manufacturing development efforts (both in-process and off-line) along with their learning strategy. Another research investigating the relationship between waste reduction and quality improvement projects with conceptual ("know-why") and operational ("know-how") learning in steel wire manufacturing, in conduced by [9]. Learning is viewed as investment on both "know-why" and "know-how" in order to contribute effectively to the reduction of waste. Analyzing data from twelve manufacturing plants, another learning model utilizing both proactive investments in quality improvement and autonomous learning-by-doing by was presented in [10].

Additionally, a framework to assess an optimal investment path between autonomous and induced learning over time in order to maximize process improvement is presented by [11]. Finally, a model to manage learning resources estimating the optimal time at which a firm should direct all its learning resources between two consecutive product generations was presented in [6].

\section{B. Manufacturing complexity}

Despite the fact that complexity has received considerable attention in a wide range of published research, it has not received much attention as an overall theory of manufacturing complexity. The reason for this is the difficulty of defining complexity since many authors agree that there is no single, generally accepted definition of complexity [12], [13].

However, the lack of a unifying measure of complexity in production systems leaves considerable room for further research. Here, the focus is set on two complexity factors intended to evaluate the relative complexity of process and design, since there is a proven record in the literature of their relationship with defective rate.

1) Process-based Complexity Factor: The process-based complexity factor is derived from a time standard defined for a set of assembly tasks. Initially proposed by [14] and utilized by [15], [16], [17] and [18] the process complexity factor $\left(C f_{P}\right)$ shows satisfactory correlation with defect rates and is defined as:

$$
C f_{P}=T M-t_{0} N_{a}
$$

where

$T M$ : the total manual assembly time,

$N_{a}$ : the number of assembly operations and

$t_{0}$ : a constant that represents a threshold minimum assembly time for the simplest assembly operation.

The Westinghouse method was used by Shibata to estimate the total assembly time for the entire product
$(T M)$ [19], while in [18] instead of using the Sony Standard Time (SST) method the Fuji-Xerox Standard Time was utilized.

2) Design-based Complexity Factor: Despite the various frameworks evaluating the simplicity and the manufacturability of a design the correlation between complexity and defective output is rarely considered in the literature. However, the following complexity factor has been empirically tested in various cases and a relationship with defective output has been evident.

The design-based complexity factor $\left(C f_{D}\right)$ is defined according to the evaluation score from the Design for Assembly Cost-Effectiveness (DAC) method, developed in 1984 by Sony Corp in Japan [20]. It is based in a DFA method for rating the ease-of-assembly and incorporates elements that are not captured by the process-based complexity factor.

$$
C f_{D}=\frac{K_{D}}{D_{i}}
$$

where

$K_{D}$ : an arbitrary coefficient for calibration with time based complexity and

$D_{i}$ : the ease of assembly calculated as the average ease-of-assembly $(d)$ for all operations $N_{a}$.

$$
D_{i}=\frac{\sum d_{i}}{N_{a}}
$$

where

$d$ : the evaluation of ease-of-assembly, according to DAC, calculated by subtracting the sum of three aspects $\left(f_{1}, f_{2}, f_{3}\right)$ of the assembly from 100 points and $N_{a}$ : number of operations.

$$
d_{i}=100 T M-\left(f_{1}+f_{2}+f_{3}\right)
$$

where

$f_{1}$ : the first aspect considers part characteristics, assessing the difficulty of acquiring and orienting a part for assembly.

$f_{2}$ : the second aspect considers assembly characteristics, assessing assembly difficulty.

$f_{3}$ : the last aspect considers base assembly characteristics.

\section{Integration of complexity factors}

Shibata was the first to integrate these complexity factors to a concrete framework in order to predict defects per unit in the semiconductor assembly. However, these two factors are indicatory and usually determined by the nature of the process. For example, in [18], after several experiments, the Shibata model proved to be inappropriate for electoromechanical products, like copiers, and the method developed in [21] was applied to assess design complexity. Also, in the same case study, the authors adapted process complexity using the Fuji-Xerox Standard Time instead of the Sony Standard Time (SST). 
This empowers the idea described by [22], that since there is not enough consistency between the standard time databases that assess complexity, the actual assembly times are all very sensitive to local assembly conditions and operator performance. Hence, it is important to integrate learning, as additional standard in a novel methodology. Current methods are using standard time values for operation times and do not take under account the learning curve behind the process. Usually average time for each operation is used, following standard work pace guidelines, that do not reflect reality.

\section{THEORETICAL FRAMEWORK}

Considering that performance improvement is not an event or a snapshot, an integrated framework on how processes progressively built results in practice is presented. A dynamic model is built upon the product realization chain that evolves along three distinct functions, the product design, the process modeling and the process execution.

\section{A. The product realization chain}

The first function is product design, during which designers explore and evaluate potentially useful concepts. During this stage, the most promising concepts are elaborated, the specifications of the product are defined, working models of design alternatives are built and prototypes of the final product are developed. Axiomatic Design, Design for $\mathrm{X}$ or Design for Manufacturing methods are used to facilitate the various aspects of new product development. Some of these tools aim to increase the product manufacturability and incorporate manufacturing issues into the design process. However, these tools usually provide design options that the manufacturing department passively follows, by translating the physical materials of the product to instructional information.

Process modeling "translates product design into technical knowledge, organizational capabilities and operating processes needed to create the product" [23, p. 33]. During this stage, process engineers address the concerns about how a new product design impacts on the execution of a process, and take initiatives to prepare for upcoming product design releases or changes. However, there is a gap in knowledge concerning process development. Usually, process development is either ignored or mixed with product development in existing empirical and theoretical reports [24]. Nevertheless the practice of decoupling product design and process development is common in many industries such as automobile assembly [25] as well as Printed Circuit Boards Assembly (PCBA) industry [26].

The last stage is the execution of the production process, when the manufacturing process is on. This phase translates all instructional information and specifications from the previous two phases into action. Consequently, the real experience of production catalyzes errors, defects or mistakes and triggers the three loops of learning that will be described in next subsection.

\section{B. The loops of learning}

The first learning loop that occurs during the execution of manufacturing process is an implementation of the learning curve law. Learning-by-doing is a concept related to repetition and practice; it is connected with the concept of autonomous learning and is developed mostly by unconscious actions of operators and engineers that observe the process repetition and fine-tune it. Practice makes perfect and with cumulative production volume, learning accelerates, repetition time is reduced, the number of defective units decreases and consequently production cost. At this point the process gradually becomes established in the plant and grows to a certain capability level, known as steady state or plateau. This learning-by-doing cycle is reported by [27] as first-order learning and by [28] as autonomous learning. Once the process has reached an initial yield, production engineers take control and conduct modifications connected with the two next two loops of learning.

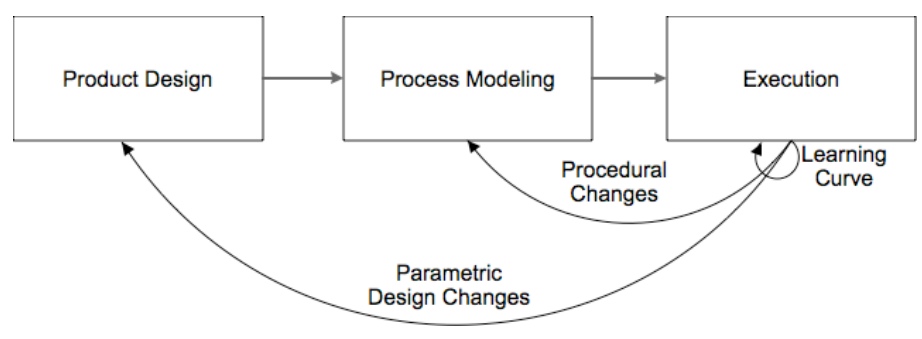

Fig. 1. Product realization chain and three loops of learning

This framework is opposite to the idea that process improvement happens concurrently with process execution, simply because improvement requires deliberate actions, time and investment to change. In [29] and [27] the extent to which performance improvement and learning is an "automatic" benefit of cumulative experience is also questioned. These conscious efforts of engineering change and organization learning, are referred in the learning literature as a part of second-order learning by [27] or induced learning by [28] and are expressed here as the two loops of procedural changes and physical changes. These loops represent the opportunity to learn and improve product performance, productivity and quality during the actual production phase. Their main difference is that in learning-by-doing loop, learning occurs inside the production system, while in the next two loops of learning, changes are made by out-of-the-box observations and interference to the system. Consequently, the last two loops of learning require not only empirical, but also analytical knowledge of the product and the process. According to the magnitude of change, temporal disruptions might occur but once the process is reestablished the yield will be higher.

The second learning loop represents the change in process practice that emerges from experience. Engineers and operators are capable of finding new or refined processes to surpass the existing level of performance, combining their creative thinking with their hands-on experience. For example, investment in process change might incorporate adjustments on 
mechanical machinery, simplified tooling, set-up optimization of a workstation, development of better and faster inspection methods or corrective actions in deviating parameters. A deviating process parameter is, for instance, the hight temperature in an oven or the low speed of a conveyor belt. These actions that determine manufactured quality are not easy tasks and researching, developing and implementing new process technology require a dynamic and active environment as it is acknowledged in [23].

The last loop occurs when, based on obtained experience, a re-design or design corrective action takes place. Design change often results from quality deviation, nonconformance, quality failure, defects, or mistakes according to [30] and [31]. Despite the inherent defect occurrence of a process, bad design or design variation are closely connected with the quality performance of a line and many defects associated with design, could not be identified before the actual production phase. There are also circumstances when the design of a product is excellent, but in practice during production there is a high potential for defects. Physical changes like size thickness or diameter of a part in order to decrease complexity and accelerate quality performance are usual during this stage. This feedback loop to parametric design changes was also reported in a ramp-up case study in Kodak camera production by [32], where the author points out that production improvements are not only results of operator learning but also design improvements.

The next section presents an approach to quantify deliberate changes that focus on quality improvement, during the learning curve utilizing the learning curve theory and complexity factors.

\section{MODEL FORMULATION}

This approach provides an aggregative view of the detailed dynamics of learning during production ramp-up, capturing the interplay between process change and product design changes. This model addresses the optimal policy of investment to change and presents an a priori estimation of final performance. In other words, attempting to bridge the gap of what is known about a production process and what needs to be known, to accelerate the learning curve and minimize defects.

This work utilize the quality learning curve of the process (defectiveness curve over time), since defective quantities (Defects Per Unit - DPU) and production loss are the practical measurements of quality. This view reflects the point that errors and defect are integral parts of learning mechanisms, that is "knowledge and error flow from the same mental sources; only success can tell the one from the other" [33]. In addition, the measure of defects not only incorporates quality assurance efforts, but also offers a better tool to explain empirical learning curve effects [34], proving that defective units are statistically more significant in quality efforts than good units.

\section{A. The learning curve}

Let $L(t)$ denote the quality learning curve of a firm when a new product enters the production phase:

$$
L(t)=a e^{-b t}+c .
$$

To address the dynamic nature of the learning rate, each time a deliberate engineering change takes place the rate of learning is modified according to the magnitude of change and the new complexity factor of the process or the product accordingly.

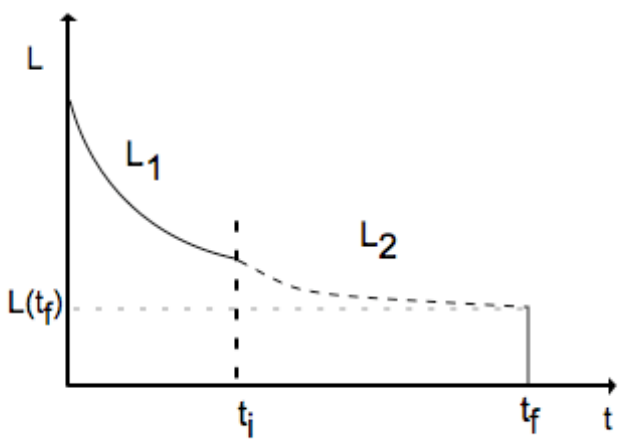

Fig. 2. Quality learning curve of the production process

Let $L_{1}(t)$ and $L_{2}(t)$ denote the learning curve before and after a deliberate engineering change respectively.

$$
\begin{aligned}
& L_{1}(t)=a_{1} e^{-b_{1} t}+c_{1} \\
& L_{2}(t)=a_{2} e^{-b_{2} t}+c_{2}
\end{aligned}
$$

The main scope is to find the optimum time point $t_{i}$ to perform a deliberate engineering change, the type of investment policy (between product design $C f_{D}$ or process modification $\left.C f_{P}\right)$ and the optimal magnitude of change $Y$ to $C f$ factor in order to minimize the target value $L\left(t_{f}\right)$ of defective level at the end of the planning horizon $t_{f}$. At any given moment of the production process this model can be applied. Giving as an input the initial complexity factors $C f_{P}$ and $C f_{D}$ and performing the dynamic algorithm for both process and product complexity factors, the optimal policy that eliminates defects is determined by minimizing $L\left(t_{f}\right)$ values.

The model is built around three state variables, the parameters of the learning curve $L_{2}$ after the engineering change $a_{2}, b_{2}$ and $c_{2}$. Both parameters $b_{2}$ and $c_{2}$ are functions of the complexity factor, while $a_{2}$ is determined according to the time of change $\left(t_{i}\right)$ and steady state parameter $c_{2}$.

$$
\begin{gathered}
b_{2}=F(F(C f)) \\
c_{2}=F(C f) \\
a_{2}=F\left(c_{2}, t_{i}\right)
\end{gathered}
$$

\section{B. Investment to change}

The initial complexity factor of a product and a process is determined during the preliminary product design and process modeling stages respectively. The change of complexity during execution has a cost analogous to the magnitude of this change. Change in product or process specifications requires serious 
investments during the ramp-up period. These investments could be financial funds, man-hours or even engineering effort. Consequently, these changes can be afforded only to a certain extend by the company.

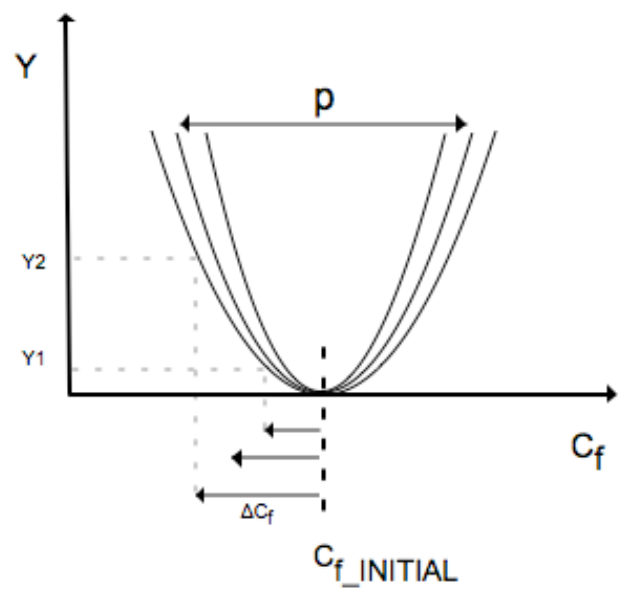

Fig. 3. Magnitude of change and cost of investment

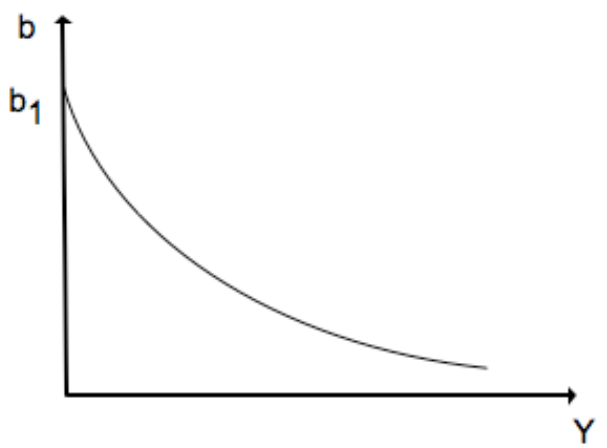

Fig. 4. Rate of learning and cost of investment

This claim can be made more explicit by considering the natural limits of change in a product or a process. For example, attempts to reduce process complexity beyond these limits might require a lot of man-hours, expensive new technology and new funds from the company. Furthermore, since process complexity is closely related with total production time and the number of operations, it could be only modified at a certain extent till the natural time limit for each operation.

On the other hand, in the case of product design a great investment in reducing its complexity factor results in the development of a new product. An oversimplification of a design, by minimizing even the functional characteristics, exceeds the limits of acceptable change and could yet result in a different product.

Figure 3 demonstrates how reductions in the complexity factor, result in increasing the cost of investment to change $(Y)$. The arrows point the direction of decrease in the complexity factor and the magnitude of change. The closer to axis-y, the more the complexity factor decreases and the cost of change increases.

Let us consider that the functional form of equation is represented by the following form:

$$
Y_{C f}=p C f^{2}+q C f+r .
$$

Since experienced engineers know empirically the limits until which are able to make changes in the production process or in the design of a product, the value of $p$ variable is considered known.

According to the form of equation 11 and the fact that its polynomial discriminant equals to zero, the state variables $r$ and $q$ can be calculated as

$$
\begin{gathered}
r=p C f^{2}, \\
q=-2 p C f .
\end{gathered}
$$

According to the complexity factor, the above equations are transformed for process complexity as:

$$
\begin{gathered}
Y\left(C f_{P}\right)=p_{p} C f_{p}{ }^{2}+q_{P} C f_{p}+r_{p} \\
r_{p}=p_{p} C f_{P}{ }^{2} \\
q_{p}=-2 p_{p} C f_{P}
\end{gathered}
$$

and for design complexity:

$$
\begin{gathered}
Y\left(C f_{D}\right)=p_{D} C f_{D}^{2}+q_{D} C f_{D}+r_{D} \\
r_{D}=p_{D} C f_{D}^{2} \\
q_{D}=-2 p_{D} C f_{D}
\end{gathered}
$$

The general premise, that "time and effort invested in a product and/or in a process design will be evident by the degree to which process execution objectives have been achieved" [24], is interpreted in this model. In this framework, the outcome of an investment in minimizing the complexity factors is reflected upon the learning rate of the process. More specifically, the rate of learning $b$ is related to the cost/amount of change $Y$ in the complexity factor and it is assumed that it follows the form:

$$
b=b_{1} e^{-\beta Y}
$$

where $b_{1}$ is the initial learning rate and $Y$ is the cost/amount of change acquired by equation 11. According to equation 20 , the rate of learning decreases at a certain pace $\beta$ when the cost/amount of change accelerates Additionally, the nature of change transforms equation 20 for process change as:

$$
b_{P}=b_{1 P} e^{-\beta_{P} Y_{P}}
$$

and for product design change as:

$$
b_{D}=b_{1 D} e^{-\beta_{D} Y_{D}} .
$$




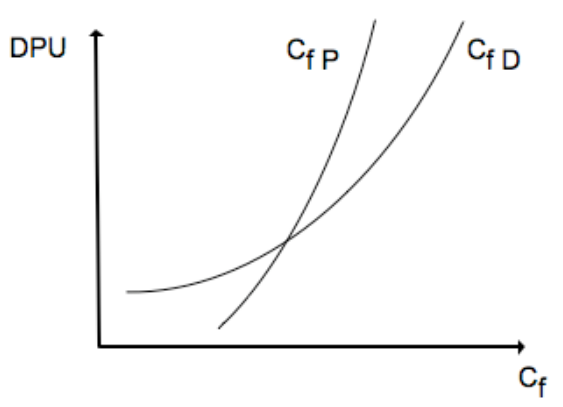

Fig. 5. Defects per unit and complexity factor

\section{Steady-state defects per unit}

Research reports a strong co-relation between the complexity factors (product and process) and Defects per Unit (DPU) [15], [35], [17], [14] [18]. These case studies focus on data of processes that had already reached a steady state. Considering that, this relationship to acquire the steady state $c$ of defect per units for both equations $L_{1}$ and $L_{2}$ is utilized.

Figure 5 presents an indicative illustration for the two complexity factors. According to previously discussed theory the steady state of a learning curve is correlated with both complexity factors. In process intensive plants DPU is affected mostly from $C f_{P}$, while in design intensive plants, DPU is affected from $C f_{D}$.

According to the empirical form of $C f_{P}$ and $C f_{D}$ presented in previous and studies, it is considered that the function of DPU and $C_{f}$ follows the form:

$$
D P U=k e^{l C f}+m,
$$

where $k, l$ and $m$ are variables calculated by the form of complexity factor over DPU.

Additionally for $C f_{P}$ :

$$
D P U_{P}=k_{P} e^{l_{P} C f_{P}}+m_{P}
$$

and for $C f_{D}$ :

$$
D P U_{D}=k_{D} e^{l_{D} C f_{D}}+m_{D}
$$

However, to continue the formulation of this model, it is assumed that both complexity factors affect DPU equally.

$$
c=\frac{D P U_{P}+D P U_{D}}{2}
$$

Consequently $c$ is a function of both complexity factors:

$$
c=F\left(C f_{P}, C f_{D}\right)
$$

To this point, the relationship between the complexity factor and parameters $b_{2}$ and $c_{2}$ has been presented. Thus, state parameters $b_{2}$ and $c_{2}$ can be expressed as function of complexity factors:

$$
\begin{gathered}
b_{2}=F(F(C f)) \\
c_{2}=F(C f)
\end{gathered}
$$

Finally, to determine factor $a_{2}$, for time $t=t_{i}$, let us consider that $L_{1}\left(t_{i}\right)=L_{2}\left(t_{i}\right)$ However, due to the fact that for $L_{2}$ the specific time slot represents its initial time slot, $t_{i}=0$.

$L_{1}\left(t_{i}\right)=L_{2}(0) a_{1} e^{-b_{1} t_{i}}+c_{1}=a_{2}+c_{2} a_{2}=a_{1} e^{-b_{1} t_{i}}+c_{1}-c_{2}$

Consequently $a_{2}=F\left(c_{2}, t_{i}\right)$.

According to the nature of change $a_{2}$ is transformed for process change as:

$$
a_{2 P}=a_{1} e^{-b_{1} t_{i}}+c_{1}-c_{2 P}
$$

and for design changes as:

$$
a_{2 D}=a_{1} e^{-b_{1} t_{i}}+c_{1}-c_{2 D}
$$

Concluding, the optimal change is specified by the minimum value $L_{2}\left(t_{f}\right)$ :

$L_{2}\left(t_{f}\right)=\min \left\{\min \left(a_{2 P} e^{-b_{2 P} t_{f}}+c_{2 P}\right), \min \left(a_{2 D} e^{-b_{2 D} t_{f}}+c_{2 D}\right)\right\}$

Figure 6 presents a flow chart of the algorithm. It can be observed that when the output is the minimum $L\left(t_{f}\right)$, a flag that indicates the optimal investment policy ( $D$ for design change or $P$ for process change), the new complexity factor $C f$ and the time of change $t_{i}$.

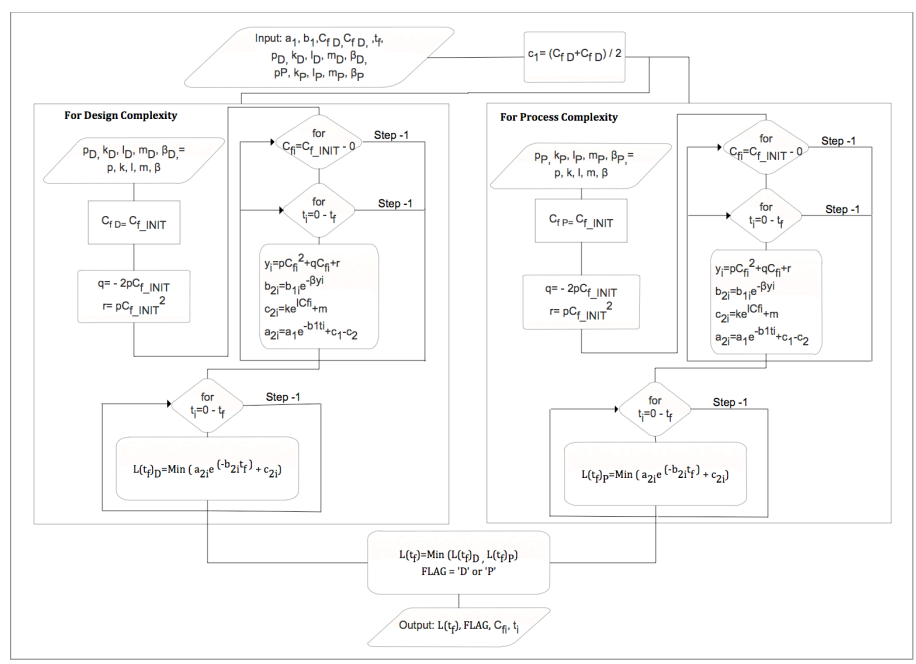

Fig. 6. Flow Chart

\section{Conclusions}

Despite the need for a concrete and useful model of learning during early development stages, a fundamental basis of the concept is missing in the literature. To fill this gap, this paper proposes a novel framework of learning during the product realization chain. This algorithm supports decision-making while the production process is on and balances between parametric design changes and procedural changes. This framework was developed based on empirical measures of complexity in order to increase the quality performance of the production process. 


\section{REFERENCES}

[1] J. D. Brooker, K. G. Swift, and N. J. Brown, "Design for assembly quality: strategies, guidelines and techniques," Journal of Engineering Design, vol. 16, no. 3, pp. 279-295, 2005.

[2] K. Swift, M. Raines, and J. D. Booker, "Analysis of product capability at the design stage," Journal of Engineering Design, vol. 10, no. 1, pp. $1-21,1999$.

[3] V. Krishnan and K. T. Ulrich, "Product development decisions: A review of the literature," Management Science, vol. 47, no. 1, pp. 1-21, 2001.

[4] J. Carrillo and R. Franza, "Investing in product development and production capabilities: The crucial linkage between time-to-market and ramp-up time," European Journal of Operational Research, vol. 171, no. 2, pp. 536-556, 2006.

[5] R. J. Urbanic and W. H. ElMaraghy, Advances in Design, ser. Springer Series in Advanced Manufacturing. Springer, 2006, vol. Chapter 35: Modeling of Manufacturing Process Complexity.

[6] L. L. Demeester and M. Qi, "Managing learning resources for consecutive product generations," International Journal of Production Economics, vol. 95, pp. 265-283, 2005.

[7] R. L. Marcellus and M. Dada, "Interactive process quality improvement," Management Science, vol. 37, no. 11, pp. 1365-1376, 1991.

[8] D. M. Upton and B. Kim, "Alternative methods of learning and process improvement in manufacturing." 16 (1), 1-20," Journal of Operations Management, vol. 16, no. 1, pp. 1-20, 1998.

[9] M. A. Lapré, A. S. Mukherjee, and L. N. V. Wassenhove, "Behind the learning curve: linking learning activities to waste reduction," Management Science, vol. 46, no. 5, pp. 597-611, 2000.

[10] C. D. Ittner, V. Nagar, and M. V. Rajan, "An empirical examination of dynamic quality-based learning models," Management Science, vol. 47, no. 4, pp. 563-578, 2001.

[11] D. A. Serel, M. Dada, H. Moskowitz, and R. D. Plante, "Investing in quality under autonomous and induced learning," IIE Transactions (Institute of Industrial Engineers), vol. 35, no. 6, pp. 545-555, 2003.

[12] A. Law and W. D. Kelton, "Simulation modeling and analysis," in 3rd edition, I. McGraw-Hill Companies, Ed., 2000.

[13] T. J. Gabriel, "Measuring the manufacturing complexity created by system design," in Southeast Decision Sciences Institute Conference, 2022 February. Orlando, Florida, 2008, pp. 614 - 649.

[14] C. M. Hinckley, "Managing product complexity, it's just a matter of time," in International Forum on DFMA: Foundations and Frontiers, June 8-10, 1998.

[15] H. Shibata, B. Cheldelin, and K. Ishii, "Assembly quality methodology: An application of the defect prediction to audio equipment assembled at various manufacturing sites," in ASME Design Engineering Technical Conference, September 9 - 12, vol. 3, 2001, pp. 333-339.

[16] H. Shibata, "Global assembly quality methodology: a new method for evaluating assembly complexities in globally distributed manufacturing," Doctoral dissertation, Stanford University, Department of Mechanical Engineering, 2002

[17] H. Shibata, B. Cheldelin, and K. Ishii, "Assembly quality method: Integrating Design for Assembly Cost-effectiveness (DAC) to improve defect prediction," in ASME International Mechanical Engineering Congress, 2 - 6 September, vol. 3, 2003, pp. 299-306.

[18] Q. Su, L. Liu, and S. Lai, "Measuring the assembly quality from the operator mistake view: a case study," Assembly Automation, vol. 29, no. 4, pp. 332-340, 2009.

[19] K. Ishii, "Design for manufacturability: Product definition," Course reader for ME217A, 1999.

[20] Y. Yamagiwa, "An assembly ease evaluation method for product designers: DAC," Techno Japan, Tech. Rep. 12, 1988.

[21] D. Ben-Arieh, "Analysis of assembly operations difficulty: a fuzzy expert system approach," Journal of Intelligent Manufacturing, vol. 4, no. 6, pp. 411-419, 1993.

[22] C. M. Hinckley, "A global conformance quality model: a new strategic tool for minimizing defects caused by variation, error, and complexity," doctoral dissertation, Department of Mechanical Engineering, Stanford University, Stanford, CA., 1993.

[23] G. Pisano, The Development Factory: Unlocking the Potential of Process Innovation. Harvard Business School Press, 1997.

[24] Q. Lu and B. Botha, "Process development: a theoretical framework," International Journal of Production Research, vol. 44, no. 15, pp. 29772996, 2006.
[25] T. Fujimoto, Comparing performance and organization of product development across firms, regions, and industries: the applicability of the automobile case, $R \& D$ Strategies in Japan: The National, Regional, and Corporate Approach. New York: Elsevier, 1993.

[26] Q. Lu and L. Wood, "The refinement of design for manufacture: inclusion of process design," International Journal of Operations \& Production, vol. 26, no. 10, pp. 1123 - 1145, 2006.

[27] P. S. Adler and K. B. Clark, "Behind the learning curve: A sketch of the learning process," Management Science, vol. 37, no. 3, pp. 267-281, 1991.

[28] F. K. Levy, "Adaptation in the production process," Management Science, vol. 11 , no. 6 , pp. B136-B154, 1965.

[29] G. P. Pisano, R. M. J. Bohmer, and A. C. Edmondson, "Organizational differences in rates of learning: Evidence from the adoption of minimally invasive cardiac surgery," Management Science, vol. 47, no. 6, pp. 752768, 2001.

[30] J. L. Burati, J. J. Farrington, and W. B. Ledbetter, "Causes of quality deviations in design and construction," Journal of Construction Engineering and Management, vol. 118, no. 1, pp. 34-49, 1992.

[31] R. Keller, C. M. Eckert, and P. J. Clarkson, "Using an engineering change methodology to support conceptual design," Journal of Engineering Design, vol. 20, no. 6, pp. 571-587, 2009.

[32] J. W. Casamento, "A model for predicting and managing a production ramp-up of a new product," Thesis (M.S.), Massachusetts Institute of Technology, Sloan School of Management, Dept. of Mechanical Engineering, 1992

[33] E. Mach, Knowledge and Error, english ed. Reidel, 1976.

[34] G. Li and S. Rajagopalan, "The impact of quality on learning," Journal of Operations Management, vol. 15, no. 3, pp. 181-191, 1997.

[35] H. Shibata, B. Cheldelin, and K. Ishii, "Assembly quality methodology: A new method for evaluating assembly complexity in globally distributed manufacturing," in ASME International Mechanical Engineering Congress, 2 - 6 September, vol. 116, 2003, pp. 335-344. 